\title{
ECOTOXICIDADE DE NANOPARTÍCULAS DE ÓXIDO DE COBRE EM MEIO AQUOSO ${ }^{1}$
}

\author{
ECOTOXICITY OF COPPER OXIDE \\ NANOPARTICLES IN AQUEOUS MEDIUM
}

\author{
Leandro Rodrigues Oviedo², Darcieli Lima Ramos', \\ Michelle Rorato Sagrilo ${ }^{3}$, Patricia Gomes ${ }^{3}$ e William Leonardo da Silva ${ }^{3}$
}

\section{RESUMO}

O extenso de uso de nanopartículas de óxido de cobre (CuO-NPs) em tratamento de água, desenvolvimento de dispositivos eletrônicos, nanomedicina, células de combustível e painéis fotovoltaicos tem resultado no descarte destas no meio aquoso. A aplicação destas nanopartículas são amplamente estudas em função de suas consideráveis propriedades ópticas, eletrônicas e funcionalidade química. Entretanto, algumas destas propriedades têm resultado em efeitos adversos aos organismos vivos da biota aquática, como indução de estresse e dano oxidativo. Neste contexto, o presente trabalho tem como objetivo apresentar uma revisão de literatura sobre CuO-NPs liberadas na água, de forma a elucidar os principais efeitos e mecanismos de ecotoxicidade causados aos organismos vivos. Com base nisso, buscou-se por artigos científicos nos indexados Scopus e PubMed, utilizando os descritores "CuO nanoparticles" AND "ecotoxicity" AND "water", segundo a Lógica Booleana. Os critérios de inclusão para a esta pesquisa foram a seleção de artigos científicos publicados nos últimos 5 anos (janeiro de 2016 a maio de 2021). Os critérios de exclusão consistiram em eliminação de artigos repetidos, revisões de literatura e artigos científicos encontrados em capítulos de livros, bem como aqueles que envolviam outra aplicação que não a avaliação de ecotoxicidade em meio aquático. Assim, foram encontrados 10 artigos científico, sendo excluídos 5 após a aplicação dos critérios de exclusão, restando apenas 5 artigos para a elaboração do presente trabalho. A partir dos resultados encontrados, foi possível verificar que as CuO-NPs são potencialmente ecotóxicas aos organismos vivos de ambiente aquático, resultando em estresse e dano oxidativo, redução da taxa de reprodução, perda da locomoção ou até mesmo morte celular. Estes efeitos estão fortemente associados ao tempo de exposição, concentração de CuO-NPs e tamanho de partícula. Portanto, sugere-se maior número de pesquisas científicas sobre o tema, bem como a elaboração de protocolos de avaliação de ecotoxicidade antes de uma etapa de transposição de escala, de escala laboratorial para industrial.

Palavras-chaves: água, sistemas nanoestruturados, nanotoxicologia.

\section{ABSTRACT}

The extensive use of copper oxide nanoparticles (CuO-NPS) in wastewater treatment, electronic devices development, nanomedicine, fuel cells and photovoltaic panels has been resulting on discharge of them on aqueous media. The application of these nanoparticles is widely studied due to considerable optic and electronic proprieties and chemical functionality. However, some of these proprieties have resulted in adverse effects to aquatic living organisms, such as induction of oxidative stress and oxidative damage. In this view, the present work aims to present a literature review about $\mathrm{CuO}-N P$ s released in water, for elucidating the main

1 Trabalho do Programa de Pós-Graduação em Nanociências.

2 Doutorandos do Programa Pós-Graduação em Nanociências. E-mail: leandro.roviedo@gmail.com; darcielilimaramos@, gmail.com

3 Professores do Programa de Pós-Graduação em Nanociências. E-mail: sagrillomr@ufn.edu.br; patriciagomes@ufn.edu.br; w.silva@ufn.edu.br 
ecotoxicological effects and mechanisms caused to living organisms. Based on that, scientific articles were searched on Scopus and PubMed indexers, using the descriptors "CuO nanoparticles" AND "ecotoxicity" AND "water", according to Boolean Logic. The inclusion criteria to this research were the selection of papers published in the last 5 years (January 2016 to May 2021). Exclusion criteria consisted in the elimination of repeated papers, literature reviews and papers found in book chapters, as well as those involving other applications different from evaluation of ecotoxicity on aqueous media. Thus, it was found 10 scientific articles, being 5 of them excluded after application of exclusion criteria, remaining 5 scientific articles for the elaboration of this work. Based on found results, it was possible to verify that CuO-NPs are potentially ecotoxic to living organisms of aquatic biota, resulting in oxidative stress and oxidative damage, reproduction rate reduction, loss of locomotion and even cell death. These effects are strongly associated to exposure time, $\mathrm{CuO-NPs}$ concentration, and particle size. Therefore, it is suggested higher development of research of this topic, as well as the development of protocols for ecotoxicological evaluation before a scale-up step, from laboratorial to industrial scale.

Keywords: water, nanostructured systems, nanotoxicology.

\section{INTRODUÇÃO}

Nanopartículas de óxidos metálicos têm sido amplamente aplicadas em tratamento de água, processos catalíticos, aplicações biomédicas, na nanomedicina e no desenvolvimento de dispositivos eletrônicos, devido, principalmente, a sua estabilidade físico-química, propriedades ópticas, eletrônicas e funcionalidade química (GOTTSCHALK; SUN; NOWACK, 2013). No entanto, o extenso uso destas nanopartículas resulta em seu descarte inadequado em corpos hídricos receptores, exercendo efeitos adversos aos organismos aquáticos e gerando grande preocupação pela comunidade científica (LOWRY et al., 2012).

As nanopartículas de óxido de cobre (CuO-NPs) possuem forte aplicação em tratamento de água, inibição de microrganismos, células de combustíveis e painéis fotovoltaicos, visto que se caracterizam por sua estabilidade química, elevada área superficial, baixa energia de band gap (1,2 - 1,7 eV), bem como suas boas propriedades eletrônicas e atividade antimicrobiana (SARAVAN et al., 2013).

As CuO-NPs podem ser sintetizadas a partir de métodos físico-químicos como síntese hidrotérmica, sol-gel ou por meio de síntese verde, também denominada biossíntese ou síntese biogênica (BENHAMMADA; TRACHE, 2021). O método hidrotérmico consiste em dissolver precursores de cobre, que são estáveis em condições hidrotérmicas e no aquecimento da mistura em sistema fechado, com ou sem direcionador orgânico (AZIMIRAD; SAFA; AKHAVAN, 2015). Enquanto, a síntese verde consiste na utilização de extratos de plantas (Malva Sylvestris, Calotropis Procera e Acalypha Indica) como agente redutor do cobre (GANESAN et al., 2020). De modo geral, as sínteses verdes de $\mathrm{CuO}$-NPs são preferíveis à síntese hidrotérmica, uma vez que requer menor demanda de energia, precursores relativamente baratos e menores tempos de reação química (ANJU et al., 2020).

Entretanto, estes sistemas nanoestruturados se baseiam em protótipos de escala laboratorial e planta-piloto, necessitando de estudos preliminares sobre sua ecotoxicidade em 
ambiente aquático anterior à etapa de transposição de escala, isto é, de laboratorial para industrial (LAZAREVA.; KELLER, 2014).

A ecotoxicidade de CuO-NPs a organismos aquáticos tem sido relatada como um adversidade ambiental, podendo prejudicar o equilíbrio ecológico da biota aquática, visto que estas nanopartículas se acumulam em sedimentos, liberando considerável parcelas de íons cobre. Entretanto, essa ecotoxicidade depende da qualidade da água, tipo de CuO-NPs (verde ou química) e das condições de exposição (temperatura, concentração de CuO-NPs e tempo), respectivamente (XIAO et al., 2016). Além disso, o tamanho da nanopartícula se caracteriza como outro fator responsável pelos efeitos tóxicos em ambiente aquático, uma vez que estas conseguem penetrar nos compartimentos corporais dos organismos vivos, os quais não são penetráveis por partículas maiores em macroescala (BONDARENKO et al., 2013).

Deste modo, ecotoxicidade aguda de CuO-NPs é reportada para animais aquáticos como algas e crustáceos, em menor extensão e para peixes em maior extensão (RAMSKOV et al., 2015). Além disso, diversos estudos de nanotoxicidade sobre animais aquáticos vertebrados e invertebrados têm sido realizados de modo a elucidar os efeitos adversos ocasionados pela liberação de CuO-NPs residual nestas águas (PRADHAN et al., 2012). Frequentemente, estes ensaios são conduzidos com espécies padrões como cladocerans D. magna e Ceriodaphia dubia, peixe Danio rerio (peixe-zebra) e Oncorhynchus mykiss (truta arco-íris), entre outros (YOO-IAM; CHAICHANA; SATAPANAJARU, 2014).

Neste contexto, o presente trabalho tem como objetivo elencar os principais avanços nos estudos de ecotoxicidade de CuO-NPs em meio aquoso como etapa preliminar à transposição de escala de processos envolvendo a aplicação destas nanopartículas. Além disso, investigar quais são os principais fatores associados à ecotoxicidade de $\mathrm{CuO}-\mathrm{NPs}$ em meio aquático e elucidar os principais mecanismos de ecotoxicidade destas nanopartículas.

\section{MATERIAIS E MÉTODOS}

Esse estudo se trata de uma pesquisa bibliográfica de caráter exploratório e de abordagem quantitativa, a fim de proporcionar o acesso às informações sobre os mecanismos e efeitos observados nos estudos de ecotoxicidade de CuO-NPs. Nesse contexto, buscou-se por artigos científicos nos indexadores Scopus e PubMed, utilizando os descritores "copper oxide nanoparticles" AND "ecotoxicity” AND “water”, segundo a Lógica Booleana. A busca foi realizada com restrição de tempo (entre janeiro de 2016 e maio de 2021). Os critérios de exclusão consistiram em estudos em andamento, capítulos de livro, trabalhos duplicados nas bases de dados e trabalhos que tangenciam o tema, bem como aqueles que não estavam diretamente relacionados ao tema. 


\section{RESULTADOS E DISCUSSÃO}

\section{RESULTADOS}

A Figura 1 apresenta o número de artigos científicos publicados no período de janeiro de 2016 a maio de 2021 pelas plataformas Scopus e PubMed. No indexador Scopus foram encontrados 7 artigos e aplicando os critérios de exclusão, exclui-se 2 artigos por se repetirem nos dois indexadores ou não se adequarem ao objetivo desta pesquisa. Paralelamente, no indexador PubMed foram encontrados 5 artigos, sendo todos excluídos após a aplicação dos critérios de exclusão (trabalhos repetidos em relação ao indexador Scopus). Deste modo, utilizou-se 5 artigos científicos para a construção deste trabalho.

Figura 1 - Número de publicações de artigos científicos no período de janeiro de 2016 a maio de 2021.



Fonte: Construção do autor.

De acordo com a Figura 1, observa-se que houve um aumento do número de publicações envolvendo o estudo da ecotoxicidade de CuO-NPs em meio aquoso no indexador Scopus, quando comparado ao indexador PubMed. No qual denota-se a escassez de pesquisas realizadas entre o período de 2019 a 2021. A partir dos resultados encontrados na plataforma Scopus, verifica-se a importância da avaliação ecotoxicológica de $\mathrm{CuO}-\mathrm{NPs}$ liberadas em meio aquoso.

\section{DISCUSSÃO}

A Tabela 1 apresenta os principais resultados encontrados para a avaliação de ecotoxicidade de $\mathrm{CuO}-\mathrm{NPs}$ em meio aquoso, em que diferentes espécies de caracóis, algas e bactérias foram estudadas como organismos-alvo da pesquisa, assim como algumas espécies de plantas. 
Tabela 1 - Estudos da ecotoxicidade de CuO-NPs em meio aquoso publicados entre os períodos janeiro de 2016 e maio de 2021.

\begin{tabular}{|c|c|c|c|c|c|c|c|}
\hline Pesquisa & Aspectos metodológicos & $\begin{array}{c}\text { Método } \\
\text { de síntese }\end{array}$ & [CuO-NPs] & $\begin{array}{l}\text { Tempo de } \\
\text { exposição }\end{array}$ & $\begin{array}{c}\text { Tamanho } \\
\text { (nm) }\end{array}$ & Dano associado & Referência \\
\hline $\begin{array}{l}\text { Estudo da ecotoxicidade } \\
\text { de CuO-NPs em diferentes } \\
\text { espécies marinhas }\end{array}$ & $\begin{array}{l}\text { Estudos com B. plicatilis } \\
\text { (rotífero), A. francisca- } \\
n a \text { (camarão), T. fulvus } \\
\text { (copépodes) e P. lividus } \\
\text { (ouriço-do-mar) }\end{array}$ & Sol-gel & $1-80 \mathrm{mg} \mathrm{L}^{-1}$ & $\begin{array}{l}2-96 \\
\text { horas }\end{array}$ & 12 e 50 & $\begin{array}{l}\text { Mortalidade, falha } \\
\text { na locomoção e } \\
\text { na fertilidade do } \\
\text { organismos vivos }\end{array}$ & $\begin{array}{l}\text { Rotini et al. } \\
\text { (2018) }\end{array}$ \\
\hline $\begin{array}{l}\text { Estudo do efeito da solubili- } \\
\text { dade e do tamanho de partí- } \\
\text { cula sobre a ecotoxicidade de } \\
\text { CuO-NPs em meio aquoso }\end{array}$ & $\begin{array}{l}\text { Determinação } \mathrm{EC}_{50} \mathrm{e} \\
\text { relação concentração-efeito } \\
\text { para a verificação da con- } \\
\text { tribuição de CuO-NPs na } \\
\text { ecotoxicidade aquática }\end{array}$ & Sol-gel & $45 \mu \mathrm{g} \mathrm{L}^{-1}$ & $7-28$ dias & 40 & $\begin{array}{l}\text { Estresse oxidativo } \\
\text { ( } 7-14 \text { dias) e dano } \\
\text { oxidativo ( } 28 \text { dias) }\end{array}$ & $\begin{array}{c}\text { Ma et al. } \\
\text { (2017) }\end{array}$ \\
\hline $\begin{array}{l}\text { Estudo dos efeitos combi- } \\
\text { nados de } \mathrm{ZnO}-\mathrm{NPs} \text { e } \mathrm{CuO} \text { - } \\
\text {-NPs para a ecotoxicidade }\end{array}$ & $\begin{array}{l}\text { Determinação } \mathrm{EC}_{50} \mathrm{e} \\
\text { relação concentração-efeito } \\
\text { para a verificação da con- } \\
\text { tribuição de CuO-NPs na } \\
\text { ecotoxicidade aquática }\end{array}$ & $\begin{array}{l}\text { Hidrotér- } \\
\text { mica }\end{array}$ & $\begin{array}{c}\text { 64-101 mg } \\
\mathrm{L}^{-1}(\mathrm{Cu}) \mathrm{e} \\
123-266 \mathrm{mg} \\
\mathrm{L}^{-1}(\mathrm{Zn})\end{array}$ & $\begin{array}{c}24-96 \\
\text { horas }\end{array}$ & $\begin{array}{c}25(\mathrm{Zn}) \mathrm{e} \\
40(\mathrm{Cu})\end{array}$ & ----- & $\begin{array}{l}\text { Ye et al. } \\
\text { (2017) }\end{array}$ \\
\hline $\begin{array}{l}\text { Estudo da exposição (via } \\
\text { ingestão) direta e indireta } \\
\text { de CuO-NPs por D. magna } \\
\text { em meio aquoso }\end{array}$ & $\begin{array}{l}\text { Design de experimentos } \\
\text { aplicados em } 14 \text { dias para } \\
\text { determinação de ecotoxici- } \\
\text { dade de CuO-NPs }\end{array}$ & $\begin{array}{l}\text { Síntese } \\
\text { termoplás- } \\
\text { mica }\end{array}$ & $\begin{array}{c}0,07 \mathrm{e} \\
15 \mathrm{mg} \mathrm{L}^{-1}\end{array}$ & $\begin{array}{c}24 \text { e } 48 \\
\text { horas }\end{array}$ & $>50$ & $\begin{array}{l}70 \text { e } 90 \% \text { de mor- } \\
\text { talidade da alga em } \\
\text { ambas as concentra- } \\
\text { ções de CuO-NPs }\end{array}$ & $\begin{array}{l}\text { Wu et al. } \\
\text { (2017) }\end{array}$ \\
\hline $\begin{array}{l}\text { Estudo do efeito do } \mathrm{pH} \\
\text { sobre a ecotoxicidade de } \\
\mathrm{CuO}-\mathrm{NPs} \text { em meio aquoso }\end{array}$ & $\begin{array}{l}\text { S. obliquus (alga) exposta a } \\
\text { CuO-NPs em solução aquo- } \\
\text { sa contendo ácido benzoico. }\end{array}$ & $\begin{array}{l}\text { Hidrotér- } \\
\text { mica }\end{array}$ & $\begin{array}{c}0,5 \mathrm{e} \\
1 \mathrm{mg} \mathrm{L}^{-1}\end{array}$ & 96 horas & 40 & Estresse oxidativo & $\begin{array}{c}\text { Wang et al. } \\
\text { (2016) }\end{array}$ \\
\hline
\end{tabular}

Fonte: Construção do autor.

Segundo a Tabela 1, verifica-se que todas as CuO-NPs são sintetizadas por meio de métodos químicos, como síntese hidrotérmica e sol-gel. Além disso, observa-se a predominância de nanopartículas com diâmetro médio na faixa de $12-50 \mathrm{~nm}$, o que caracteriza estes nanomateriais como mesoporosos (THOMMES et al., 2015). Ainda, nestes estudos, foi avaliada a ecotoxicidade de CuO-NPs sobre algumas espécies de organismos vivos de ambiente aquático, dentre eles crustáceos, gastrópodes e algas, principalmente. Adicionalmente, pode-se verificar que as pesquisas científicas apresentadas buscam verificar possíveis correlações entre o tamanho de partícula, a concentração de CuO-NPs, o tempo de exposição, a estabilidade físico-química e a ecotoxicidade. Com base nisso, as seções subsequentes abordam cada efeito separadamente, de forma a elucidar estas correlações e os mecanismos de ecotoxicidade associado à exposição de CuO-NPs em meio aquático.

\section{Efeito do tamanho de partícula e tempo de exposição}

Uma correlação entre o tamanho de partícula e ecotoxicidade de CuO-NPs é relatada nos artigos científicos apresentados na Tabela 1. A partir destes, é possível confirmar que todas as nanopartículas estudadas possuem estrutura mesoroporosa, com diâmetros médios reportados como 
$12,25,40$ e $50 \mathrm{~nm}$ e induzem estresse oxidativo aos animais aquáticos após curtos tempo de exposição (na ordem de 2 a 24 horas) e concentrações na faixa 10-80 $\mathrm{mg} \mathrm{L}^{-1}$. Em alguns casos, a indução de estresse oxidativo é reportada após 48 h (ROTINI et al., 2018). Assim, à medida que o tamanho de partícula aumenta (de 12 a $50 \mathrm{~nm}$ ), a ecotoxicidade diminui, visto que a energia de superfície de CuO-NPs diminui com o aumento de tamanho de partícula (maior estabilidade físico-química) e consequentemente, reduz-se a aglomeração destas (PENG et al., 2017). Além disso, as nanopartículas de maior diâmetro não conseguem acessar compartimentos corporais com a mesma facilidade que aquelas com menor diâmetro (LI et al., 2013).

Paralelamente, observa-se que independentemente do tamanho de partícula, tempos de exposição prolongados favorecem o aumento de ecotoxicidade de $\mathrm{CuO}-\mathrm{NPs}$, induzindo dano oxidativo aos organismos vivos. Isso se deve principalmente à estabilidade físico-química relativamente baixa de $\mathrm{CuO}-\mathrm{NPs}$, resultando em aglomeração das partículas ou aderência destas nas membranas celulares de organismos, promovendo ligações com proteínas e ribossomos como mecanismos de aumentar sua estabilidade físico-química (CHEN et al., 2015). Adicionalmente, estas nanopartículas se mostram passíveis de penetração em diversas barreiras corporais dos organismos vivos estudados, sendo absorvidos principalmente no intestino, no hepatopâncreas e tecido muscular, resultando em dano ao DNA, mitocondrial e até mesmo morte celular (MA et al., 2016; LI et al., 2013; NEL et al., 2006).

\section{Efeito da solubilidade}

Os artigos científicos reportados na Tabela 1 também avaliam o efeito da solubilidade $\mathrm{CuO}$ -NPs sobre a ecotoxicidade em ambiente aquático. Embora as CuO-NPs possuam baixa solubilidade em água, relata-se haver uma porcentagem de cobre que é liberada da nanoestrutura e posteriormente, dissolvida no meio aquoso (WANG et al., 2016). Deste modo, há evidências claras de que há uma contribuição do cobre dissolvido, oriundo das $\mathrm{CuO}-\mathrm{NPs}$, associado aos efeitos tóxicos, em gastrópodes, crustáceos e algas, ainda que pequena (0,16-22\%).

O cobre dissolvido, neste caso, consiste no íon $\mathrm{Cu}^{2+}$, cuja natureza química o torna passível de ligação com diversas biomoléculas livres e organelas (ribossomos), induzindo autofagia celular após certo tempo de exposição (CHEN et al., 2015). Além disso, o mecanismo de ecotoxicidade parece estar relacionado à capacidade do íon em alterar a concentração de metais no plasma (GRIFFITI et al., 2007). Em um dos trabalhos, uma forte correlação entre a redução de pH e o aumento ecotoxicidade foi encontrada, a partir da adição de ácido benzoico na solução aquosa contendo CuO-NPs. Neste caso, a contribuição de cobre dissolvido para a ecotoxicidade de CuO-NPS é relatada com a mais alta possível (22\%) (WANG; FANG; WANG, 2016), embora não seja o fator predominantemente causador dos efeitos tóxicos associados. 


\section{Efeito da energia de superfície}

Analogamente às seções anteriores, cabe analisar que os artigos científicos selecionados para estudo na presente revisão avaliam o efeito da estabilidade físico-química de CuO-NPs sobre os efeitos ecotoxicológicos causados aos organismos vivos da biota aquática. Na maioria dos trabalhos, avalia-se a estabilidade físico-química por meio da determinação de potencial zeta, onde valores (em módulo) iguais a $30 \mathrm{mV}$ indicam estabilidade devido aos fatores eletrostáticos, enquanto valores menores que 20 $\mathrm{mV}$, sugerem estabilidade em virtude dos fatores estéricos (KUZNIATSOVA et al., 2007).

Diante disso e com base nos trabalhos encontrados na literatura (Tabela 1), observa-se certa estabilidade de CuO-NPs em meio aquoso, porém com uma tendência a reduzir seu potencial zeta quando em contato com os organismos vivos, resultando em um aumento na ecotoxicidade em ambiente aquático. Para concentrações superiores a $10 \mathrm{mg} \mathrm{L}^{-1}$ expostas a gastrópodes, crustáceos e algas, relata-se redução do potencial zeta, acompanhado de estresse oxidativo induzido pelas CuO-NPs. Essa tendência pode ser explicada pela elevada energia superficial das nanopartículas, que procuram se estabilizar por aglomeração ou por combinação direta com biomoléculas livres de forma a reduzir esta energia (CHEN et al., 2015). De modo geral, o estresse oxidativo é relatado quando os valores de potencial zeta são reduzidos de $30 \mathrm{mV}$ a aproximadamente $19 \mathrm{mV}$, sugerindo baixa estabilidade físico-química das $\mathrm{CuO}-\mathrm{NPs}$.

\section{Bioacumulação de CuO-NPs em tecidos de organismos vivos}

Diversos estudos encontrados na literatura esclarecem que o destino final de CuO-NPs descartados em águas superficiais e residuárias consiste na sua deposição em sedimentos e rochas situados nos rios, lagos, mares e oceanos (RAMSKOV et al., 2015). Embora possuam baixa solubilidade em água, com o passar do tempo, CuO-NPs liberam pequenas concentrações de íons cobre $\left(\mathrm{Cu}^{2+}\right)$, que permanecem dissolvidos no meio aquoso. Ao entrar em contato de forma direta ou indireta com animais aquáticos, estas nanopartículas contribuem para os efeitos ecotoxicológicos causados nestes organismos, como estresse oxidativo, redução da taxa de reprodução, perda de locomoção e em alguns casos, ocasionando a morte (ROTINI et al., 2018). Entretanto, ainda que esta contribuição seja pequena em relação à contribuição do tamanho de partícula, energia superficial específica, tempo de exposição e concentração de $\mathrm{CuO}-\mathrm{NPs}$, esta deve ser considera em todo tipo de avaliação ecotoxicológica, preliminar a uma etapa de transposição de escala.

Com base nisso, os artigos científicos relatam que após a liberação de íons $\mathrm{Cu}^{2+}$, a absorção e a bioacumulação de cobre nos organismos vivos (gastrópodes, crustáceos e algas) ocorrem principalmente no intestino, tecido muscular, gônadas e hepatopâncreas, respectivamente (MA et al., 2016). 


\section{CONCLUSÃO}

A partir do presente estudo, verifica-se que as $\mathrm{CuO}-\mathrm{NPs}$ são potencialmente ecotóxicas, uma vez que podem induzir estresse oxidativo em curtos tempos de exposição e dano oxidativo em tempos de exposição mais prolongados. Os mecanismos de toxicidade ocorrem como resultado da absorção e bioacumulação de $\mathrm{CuO}-\mathrm{NPs}$ nos tecidos dos organismos vivos, como tecido muscular, hepático e intestino. Além, deve-se atentar ao fato de que estas nanopartículas apresentam elevada energia de superfície, o que induz sua aglomeração no meio em que se encontram. Paralelamente, verificou-se que a ecotoxicidade das $\mathrm{CuO}-\mathrm{NPs}$ aos organismos aquáticos é fortemente influenciada pelo tempo exposição, concentração de CuO-NPs e tamanho de partícula e em menor extensão, do pH do meio aquoso e do teor de cobre dissolvido. Desta forma, o aumento do tempo de exposição e da concentração, bem como a redução do tamanho de partícula podem interferir de forma significativa no crescimento, reprodução, locomoção e sobrevivência de animais aquáticos. Portanto, a partir da presente revisão de literatura, foi possível confirmar que as $\mathrm{CuO}$-NPs podem acarretar significativos efeitos ecotoxicológicos à biota aquática, necessitando-se assim um maior número de pesquisas de ecotoxicidade a fim de elucidar ainda mais os mecanismos de toxicidade e os efeitos adversos causados pelas $\mathrm{CuO}-\mathrm{NPs}$, bem como para promover/propiciar a elaboração de um protocolo adequado para um estudo de ecotoxicidade preliminar à etapa de transposição de escala laboratorial para industrial.

\section{REFERÊNCIAS}

ANJU; SHARMA, S.; DHANETIA, H. R.; SHARMA, A. Green synthesis of copper Nanoparticles using Holoptelea integrifolia fruit extract. Rasayan Journal of Chemistry, v. 13, p. 2664-2671, 2020.

AZIMIRAD, R.; SAFA, S.; AKHAVAN, O. Hydrothermally synthesized CuO powders for photocatalytic inactivation of bacteria. Acta Physica Polonica A, v. 127, n. 6, p. 1727-1731, 2015.

BENHAMMADA, A.; TRACHE, D. Green synthesis of $\mathrm{CuO}$ nanoparticles using malva sylvestris leaf extract with different copper precursors and their effect on nitrocellulose thermal behavior. Journal of Thermal Analysis and Calorimetry, 2021.

BONDARENKO, O.; JUGANSON, K.; IVASK, A.; KASEMETS, K.; MORTIMER, M.; KAHRU, A. Toxicity of $\mathrm{Ag}, \mathrm{CuO}$ and $\mathrm{ZnO}$ nanoparticles to selected environmentally relevant test organisms and mammalian cells in vitro: a critical review. Archives of Toxicology, v. 87, p. 1181-1200, 2013. 
GANESAN, K.; JOTHI, V. K.; NATARAJAN, A.; RAJARAM, A.; RAVICHANDRAN, S.; RAMALINGAM, S. Green synthesis of copper oxide nanoparticles decorated with graphene oxide for anticancer activity and catalytic applications. Arabian Journal of Chemistry, v. 13, p. 6802-6814, 2020.

GOTTSCHALK, F.; SUN, T.; NOWACK, B. Environmental concentrations of engineered nanomaterials: review of modeling and analytical studies. Environmental Pollution, v. 181, p. 287-300, 2013.

GRIFFITT, R. J.; WEIL, R., HYNDMAN, K. A.; DENSLOW, N. D.; POWERS, K.; TAYLOR, D.; BARBER, D. S. Exposição a nanopartículas de cobre causa lesão branquial e letalidade aguda em zebrafish (Danio rerio). Environmental Science \& Technology, v. 41, p. 8178-8186, 2007.

KUZNIATSOVA, T.; KIM, Y.; SHQAU, K.; DUTA, P.; VERWEIJ, H. Zeta potential measurements of zeolite Y: Application in homogeneous deposition of particle coatings. Microporous and Mesoporous Materials., v. 103, p. 102-107, 2007.

LAZAREVA, A.; KELLER, A. A. Estimating potential life cycle releases of engineered nanomaterials from wastewater treatment plants. ACS Sustainable Chemistry \& Engineering, v. 2, p. 1656-1665, 2014.

LI, K.; ZHAO, X.; HAMMER, B. K.; DU, S.; CHEN, Y. Nanoparticles inhibit DNA replication by binding to DNA: Modeling and experimental validation. ACS Nano, v; 7, p. 7:9664-9674, 2013.

LOWRY, G. V.; GREGORY, K. B.; APTE, S. C.; LEAD, J. R. Transformations of nanomaterials in the environment. Environmental Science \& Technology, v. 46, p. 6893-6899, 2012.

NEL, A.; XIA, T.; MADLER, L.; LI, N. Toxic potential of materials at the nanolevel. Science. v. 311 , p. $622-627,2006$.

PENG, C.; SHEN, C.; ZHENG, S.; WANG, W.; HU, H.; LIU, J.; SHI, J. Transformation of CuO nanoparticles in the aquatic environment: influence of $\mathrm{pH}$, electrolytes and natural organic matter. Nanomaterials (Basel), v 7, p. 326-342, 2017.

PRADHAN, A.; SEENA, S.; PASCOAL, C.; CÁSSIO, F. Copper oxide nanoparticles can induce toxicity to the freshwater shredder Allogamus ligonifer. Chemosphere, v. 89, p. 1142-1150, 2012. 
RAMSKOV, T.; CROTEAU, M.-N.; FORBES, V. E.; SELCK, H. Biokinetics of different-shaped copper oxide nanoparticles in the freshwater gastropod, Potamopyrgus antipodarum. Aquatic Toxicology, v. 163, p. 71-80, 2015.

ROTINI, A.; GALLO, A.; PARLAPIANO, I.; BERDUCCI, M. T.; BONI, R.; TOSTI, E.; PRATO, E.; MAGGI, C.; CICERO, A. M.; MIGLIORE, L.; MANFRA, L. Insights into the CuO nanoparticle ecotoxicity with suitable marine model species. Ecotoxicology and Environmental Safety, v. 147, p. 852-860, 2018.

SARAVANAN, R.; KARTHIKEYAN, S.; GUPTA, V.; SEKARAN, G.; NARAYANAN, V.; STEPHEN, A. Enhanced photocatalytic activity of $\mathrm{ZnO} / \mathrm{CuO}$ nanocomposite for the degradation of textile dye on visible light illumination. Materials Science and Engineering: C, v. 33, n. 1, p. 91-98, 2013.

THOMMES, M; KANEKO, K; NEIMARK, A. V; OLIVIER, J. P; REINOSO, F. R; ROUQUEROL, J; SING, K. S. W. Physisorption of gases, with special reference to the evaluation of surface area and pore size distribution (IUPAC Technical Report). Pure and Applied Chemistry, v. 87, p. 1051-1069, 2015.

WANG, Z.; CHEN, J. W.; LI, X.; SHAO, J. P.; PEIJNENBURG, W. J. G. M. Aquatic toxicity of nanosilver colloids to different trophic organisms: contributions of particles and free silver ion. Environmental Toxicology and Chemistry, v. 31, p. 2408-2413, 2012.

WANG, Z.; FANG, H.; WANG, S. Benzoic acid interactions affect aquatic properties and toxicity of copper oxide nanoparticles. Bulletin of Environmental Contamination and Toxicology, v. 97 , p. 159-165, 2016.

XIAO, Y.; PEIJNENBURG, W. J.; CHEN, G.; VIJVER, M. G. Toxicity of copper nanoparticles to daphnia magna under different exposure conditions. Science of The Total Environment, v. 563-564, p. 81-88, 2016.

YOO-IAM, M.; CHAICHANA, R.; SATAPANAJARU, T. Toxicity, bioaccumulation and biomagnification of silver nanoparticles in green algae (Chlorella sp.), water flea (Moina macrocopa), blood worm (Chironomus spp.) and silver barb (Barbonymus gonionotus). Chemical Speciation \& Bioavailability, v. 26, p. 257-265, 2014. 